\title{
Prototype "Electron Lens" Set-up for the Tevatron Beam-Beam Compensation
}

\author{
C.Crawford, F.Niell, G.Saewert, J.Santucci, A.Sery, A.Shemyakin*, V.Shiltsev, D.Wildman, FNAL \\ A.Aleksandrov, L.Arapov, G.Kuznetsov, P.Logachov, A.Sharapa, B.Skarbo, B.Sukhina, Budker INP
}

\section{Abstract}

A prototype "electron lens" for the Tevatron beam-beam compensation project is commissioned at Fermilab. We describe the set-up, report results of the first tests of the electron beam, and discuss future plans.

\section{SCOPE}

Compensation of beam-beam effects in the Tevatron with electron beams is a promising technique to improve the collider performance [1]. The method implies that an antiproton beam propagates through a countertraveling low-energy high-current electron beam ("electron lens"). An impact of the negative electron space charge can reduce betatron tune spread within antiproton bunch and a bunch-to-bunch tune spread - the effects due to collision with intensive proton beam which limit beam lifetime and luminosity.

An experimental R\&D program on beam-beam compensation was started at FNAL Beam Division early in 1998. The "electron lens" prototype has been designed, fabricated, assembled in the Linac Lab and commissioned in December 1998. The goal of the set-up is to study feasibility and properties of the electron beam required for the beam-beam compensation. Currently, these studies are under way.

Table 1 shows the Tevatron "electron lens" (TEL) design parameters and parameters of the prototype set-up operation to date.

\begin{tabular}{llcc} 
Parameter & units & TEL & Prototype \\
\hline Effective length, & $\mathrm{m}$ & 2.0 & 1.96 \\
Electron current,max, & $\mathrm{A}$ & 2.2 & 3.05 \\
Electron energy, max & $\mathrm{kV}$ & $5-10$ & 6.4 \\
CW modulation time, & $\mu \mathrm{s}$ & $0.4(0.13)$ & 0.5 \\
Solenoid field, & $\mathrm{kG}$ & 50 & 4 \\
Beam deviousness,rms & $\mathrm{mm}$ & 0.1 & 0.07 \\
Configuration & & 2 bends & straight \\
Beam shape control & & yes & yes
\end{tabular}

\section{2 "ELECTRON LENS" PROTOTYPE}

Major components and systems of the "electron lens" prototype are: electron gun, electron collector, modulator, power supplies, magnetic system, vacuum system, control system. The set-up (in present configuration) is shown in Fig.1. Electrons are thermally emitted from a cathode of the gun and extracted toward the positive potential of an anode $U_{a}$. Then they propagate through some $2 \mathrm{~m}$ long beam pipe which is under potential $U_{p}$ (usually, $U_{p} \approx U_{a}$ ). Finally, electron beam is absorbed in a high efficiency collector at a smaller potential $U_{c} \leq U_{a}$. Some of the electrons can be

\footnotetext{
${ }^{*}$ on leave from BINP, Novosibirsk, Russia
}

lost and absorbed on the vacuum pipe walls or at other aperture limits, i.e., not in the designated place of the collector. Strong longitudinal magnetic fields (of the order of few $\mathrm{kG}$ all along the set-up) helps to keep these losses low.

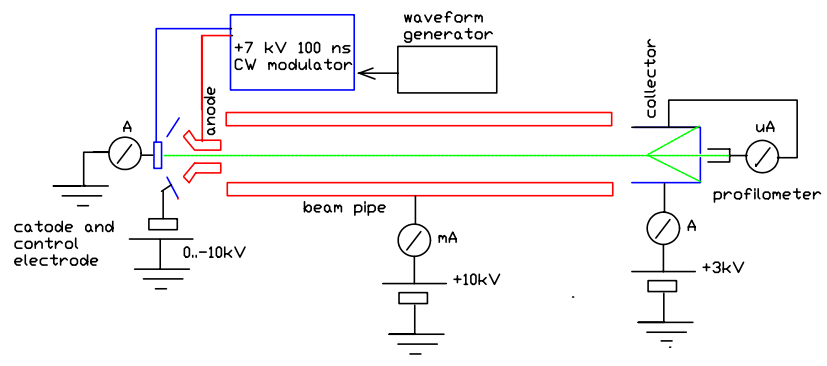

Figure 1: Electrical scheme of the "electron lens" prototype.

The high perveance electron gun is made in accordance with a novel approach proposed in [2] based on use of a convex cathode. The gun is immersed in $0.7-2 \mathrm{kG}$ longitudinal magnetic field. It has a uniform current density profile, low transverse beam temperature and high perveance (these conditions can not be met in a standard Pierce geometry with a planar cathode). Fig. 2 shows the gun construction.

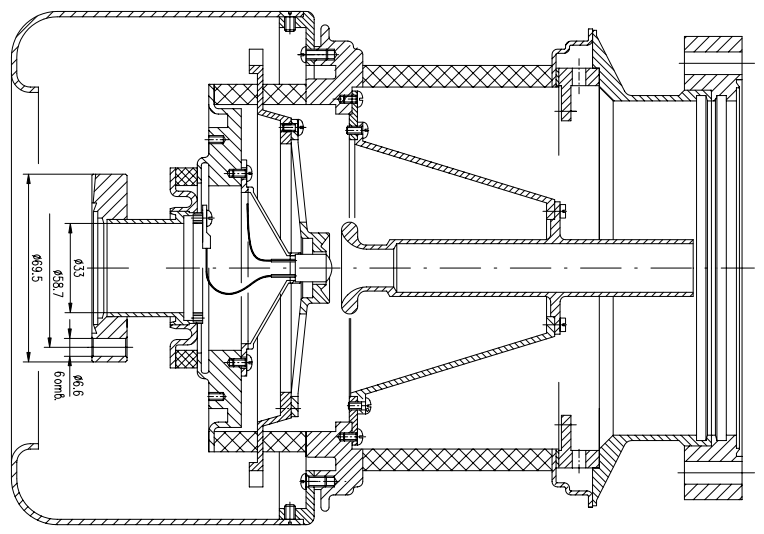

Figure 2: Electron gun of the "electron lens"

A $10 \mathrm{~mm}$ diameter $45^{\circ}$ convex cathode is made of tungsten impregnated with emitting oxides (made by HeatWave Co., CA; see details in [3]). Fig.3 shows the maximum electron current $J_{e}$ vs voltage between the anode and the cathode of the gun $U_{a}$. The maximum current is limited by negative space charge of the electron cloud near the cathode and follows Child's law $J_{e}=\mathcal{P} U_{a}^{3 / 2}$. Numerical simulations of the gun yielded the perveance $\mathcal{P}=4.9$. $10^{-6} A / V^{3 / 2}$, while a fit of the measured current at the Fig.3 gives somewhat larger perveance of $\mathcal{P}=5.85$. $10^{-6} A / V^{3 / 2}$. 


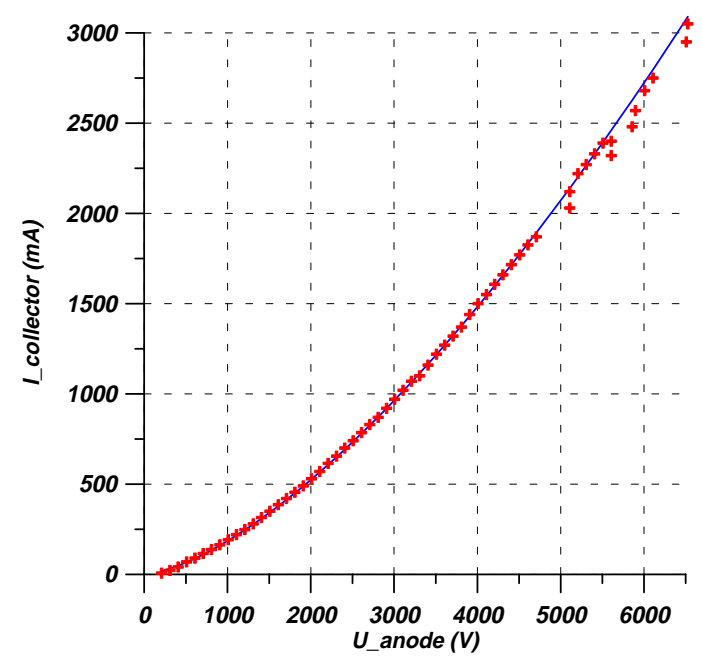

Figure 3: Current in the collector vs anode voltage. Smooth line represents a fit accordingly to Child's law with microperveance of $\mu \mathcal{P}=5.85$.

A possible explanation of excessive perveance (and current) can be imperfect alignment of the cathode and a control electrode. The latter is a specially shaped ring (simulated by computer) placed around the cathode which can vary the electron beam profile depending on its potential relative to the cathode. If the control electrode is grounded as well as the cathode $U_{c e}=0$, then the electron beam current profile has a smoothed rectangular shape. If $U_{c e}$ is negative and more than 3.5 times the positive anode potential, $-U_{c e} \geq 3.5 \cdot U_{a}$, then no current comes from the cathode (full emission suppression). At the intermediate cases, the negative control electrode potential $0<-U_{c e}<3.5 \cdot U_{a}$ suppresses the emission from the areas of the cathode which are close to the electrode, the total current is reduced and the profile becomes "bell-shape-like". Fig.4 demonstrates an example of such a shape when the total current is reduced on about $30 \%$ from $0.22 \mathrm{~A}$ (at $U_{c e}=0$ ) to $0.16 \mathrm{~A}$ (other parameters: $B_{\text {gun }}=2 \mathrm{kG}, B_{\text {coll }}=0.5 \mathrm{kG}$, main solenoid field $2 \mathrm{kG}, U_{a}=1.9 \mathrm{kV}, U_{\text {coll }}=2.5 \mathrm{kV}, U_{\text {pipe }}=2.5 \mathrm{kV}$ ).

The electron beam current profile measurements are possible using a profile analyzer installed in the collector. The collector is able to absorb about $5-10 \mathrm{~kW}$ of electron beam power on its water cooled walls. There are no additional electrodes in the collector for repelling secondary electrons (low energy electrons born after the incoming electrons hit the collector walls) and reflected part of the primary beam. To lower the outcoming stream of electrons, the collector is designed to work in a magnetic field quickly decreasing from about $2 \mathrm{kG}$ at the collector entrance to almost zero at its backplate. Good collector efficiency allowed us to achieve very small relative losses of electrons - typically of the order of $5 \cdot 10^{-4}$ (minimum $10^{-4}$ ). The losses were of extreme concern at the stage of commissioning because a) they caused intensive outgassing from the vacuum pipe wall, b) there was a fear of excessive heat release at the anode (if the electron current goes onto it), c) beam pipe potential was kept by a high voltage power supply with maximum current of $2.5 \mathrm{~mA}$ and that sets a limit on maximum allowable losses. Routinely, positive potential at the collec- tor is 1.5-2 times less than the anode potential and that is enough to keep the losses low.

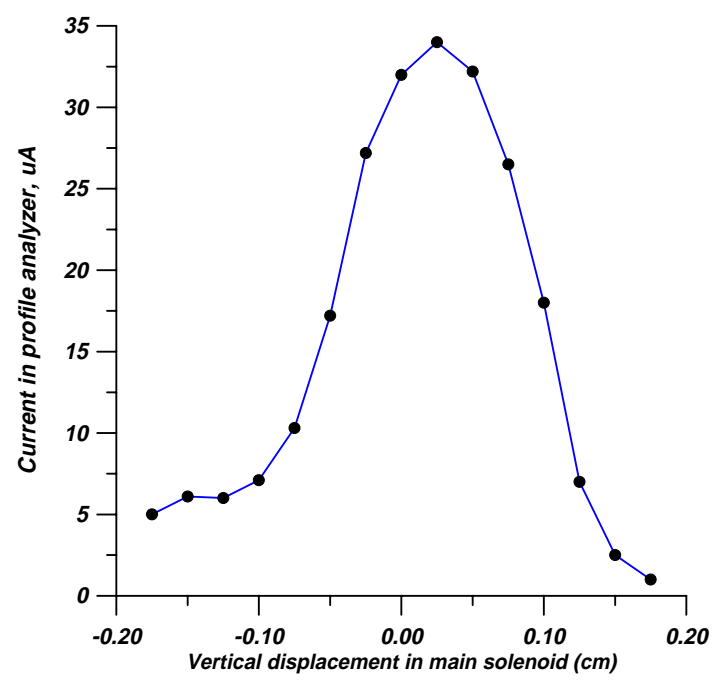

Figure 4: Vertical beam scan: analyzer current vs beam deflection in main solenoid.

The backplate of the collector has a tiny hole $0.2 \mathrm{~mm}$ in diameter and an additional Faraday cup behind it to measure the current which goes through the hole. This current is a measure of the electron beam current density. Using dipole correctors we are able to move the electron beam across the hole and measure the current profile like one presented in Fig.4. Usually a magnetic field $B_{\text {coll }}$ of a few hundreds Gauss helps to increase the current density (as the adiabatic invariant in the magnetic field is $B a^{2}=$ const, $a$ is electron beam size) and, thus, the current coming through the hole, to easily detectable level of dozens of $\mu \mathrm{A}$.

The magnetic system of the "electron lens" prototype consists of three solenoid magnets, 17 dipole correctors and independent power supplies. Two solenoid magnets $(50 \mathrm{~cm}$ long and $28 \mathrm{~cm}$ inner diameter) produce longitudinal magnetic fields in the gun and collector $(4 \mathrm{kG}$ maximum with $2.7 \mathrm{kA}$ of the coil current, typical values are $1 \mathrm{kG}$ in the gun and 0 in the collector). The main solenoid (1.96 $\mathrm{m}$ long, 20 $\mathrm{cm}$ inner diameter) provides $4 \mathrm{kG}$ with $10 \mathrm{kA}$ of the coil current. One of the goals of the prototype is to get a straight electron beam in the main solenoid with a deviation less than $0.1 \mathrm{~mm}$ rms. Because low temperature electrons in a strong magnetic field just follow the magnetic field lines, we paid special attention to the magnetic field quality. The ways to keep the field distortions low are a) precise coil fabrication and winding, b) special measures to distribute evenly the return current in 8 rods of the main solenoid, c) dipole corrector coils to compensate the field imperfections on axis of the main solenoid, d) a magnetic shield over the magnets. With these steps we have achieved the field errors $\delta B_{\perp} / B_{\|}$of about $10^{-4}$ in the main solenoid, about $10^{-3}$ in the central region of the gun and collector solenoids, and about 0.01 in the gap between the main and gun solenoid magnets. Most of imperfections take place either near the ends of the magnets, or in the middle of the main solenoid, where two of its sections are connected together (see details 
in [4]). The resulting deviousness of the magnetic field lines (and, therefore, the electron beam) is about $0.05 \mathrm{~mm} \mathrm{rms}$ in each transverse plane, or about $0.07 \mathrm{~mm}$ total. The vacuum pipe, gun and collector were installed into the solenoids only after the measurements and correction of the magnetic field were completed.

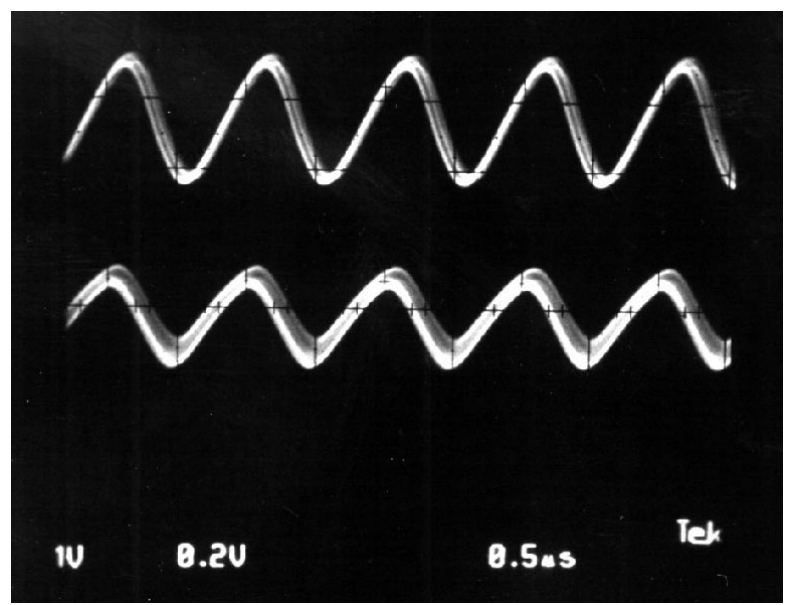

Figure 5: Current modulation in the "electron lens" prototype with frequency $1 \mathrm{MHz}$. Upper line - current in the collector $0.2 \mathrm{~A} / \mathrm{div}$ (0.43 A peak-to-peak), lower line - anode voltage $1 \mathrm{kV} / \mathrm{div}$ ( $1.5 \mathrm{kV}$ peak-to-peak).

A linear "electron lens" will be used to compensate bunch-to-bunch tune spread in the Tevatron antiproton beam. The minimum bunch spacing in the Tevatron, that is $396 \mathrm{~ns}$ in the Run II and $132 \mathrm{~ns}$ in the TEV33 upgrade of the collider. A straightforward way to get needed $\mathrm{CW}$ modulation of the current is to vary the anode voltage $U_{a}(t)$. The anode is modulated using a grid driven, $25 \mathrm{~kW}$ tetrode (EIMAC 4CW25000B) in series with a $5 \mathrm{kOhm}$ resistor connected between the tetrode's plate and the $10 \mathrm{kV}, 1.5 \mathrm{~A}$ DC plate power supply. The anode is attached directly to the plate of the tetrode resulting in the $70 \mathrm{pF}$ anode capacitance being added in parallel with the $25 \mathrm{pF}$ tube output capacitance. The tetrode is operated in a grounded cathode configuration with the grid being driven by a $150 \mathrm{~W}$ solidstate amplifier. A $60 \mathrm{~Hz}$ de-rippling circuit is connected in series with the DC grid bias to remove any unwanted 60 $\mathrm{Hz}$ modulation due to the AC filament supply. Fig.5 shows $500 \mathrm{~ns}$ modulation of the anode voltage $(1.5 \mathrm{kV}$ peak-topeak, $3 \mathrm{kV}$ maximum)- see lower line, and the total electron current ( 0.43 A peak-to-peak, 0.7 A maximum) - see upper line. Future upgrades to the modulator include lowering the plate series resistance and obtaining a higher current DC plate supply. These two upgrades will allow a greater degree of anode modulation at higher frequencies. A new all solid-state modulator, utilizing MOSFET technology, is also under development [5].

The "electron lens" control system utilizes Internet Rack Monitors (IRMs) for all of its settings, read backs, control and status. The IRM is a general purpose intelligent data acquisition system based on the VME chassis and the
Motorola MVME-162 processor. It contains the processor, network connection and the analog and digital $\mathrm{I} / \mathrm{O}$ in the same chassis. The IRM's capabilities are expandable via two VME slots. As its name suggests, this device is a node on the world-wide Internet. Experimenters can access the IRM via any terminal with permission and the right interfacing software (LabVIEW, FNAL's "Local Station", etc.) A local database within each IRM allows it to function as a stand-alone control system for the equipment it controls.

High vacuum during routine operation is provided by a diode ion pump with $50 \mathrm{l} / \mathrm{s}$ rate. A turbomolecular pump with liquid nitrogen trap is used for rough pumping and during baking. A T-crossing located in a gap between the main solenoid and the gun solenoid provides a connection of the main pipe with the vacuum pumps. The entire vacuum system was assembled and baked at $250-300^{\circ} \mathrm{C}$ over few days. The main concern during the system commissioning was outgassing of the internal surfaces of the collector and the vacuum pipe due to currents of primary and secondary electrons. The beam current was increased step by step, keeping the vacuum below $10^{-6}$ Torr (in order to to reduce the cathode emission capability) and the electron current to the pipe less than $2.5 \mathrm{~mA}$. Continuous increase of the produced electron charge in the system resulted in steady vacuum improvement. Finally, we achieved the design goal of $2 \mathrm{~A}$ on December 23, 1998. A month later, after installation of an additional collector power supply, we got more than 3 A of electron current. At the present time, the vacuum without the electron beam is about $2 \cdot 10^{-9}$ Torr, while 1 A current leads to pressure of $(3-5) \cdot 10^{-8}$ Torr. The set-up operation has become easy and very reliable. It takes about ten minutes (mostly to heat the cathode) to get a high current electron beam.

Our future plans at the "electron lens" prototype include studies of the collector efficiency vs the set-up parameters, electron beam profile measurements with a thin wire in main solenoid; and installation of diagnostic tools to control the amount of ions and secondary electrons in the system. A new (smaller size) electron gun and collector are under consideration.

Our sincere thanks to Prof.L.Tecchio (INFN, Padova, Italy) for lending two short solenoid magnets. We are grateful to A.Makarov, S.Nagaitsev, A.Warner, D.Wolff and V.Yarba for valuable contribution to the set-up design. We acknowledge technical assistance of L.Bartelson, A.Chupira, D.Douglas and B.Ogert.

\section{REFERENCES}

[1] V.Shiltsev, et.al, "Compensation of Beam-Beam Effects in the Tevatron with Electron Beams", FNAL-Pub-98/260 (1998); see also these Proceedings.

[2] A.Sharapa, et.al, NIM A, 406 (1998), p.169.

[3] J.L.Cronin, Microwave Journal, Sept. 1979, p. 57

[4] C.Crawford, et.al, these Proceedings.

[5] F.Niell, "Electron Gun Beam Extraction with MOSFETS ", this Proceedings. 